\title{
EFECTO DE LA INCLUSIÓN DE CEBADA GRANO Y SEMILLA DE GIRASOL EN UNA DIETA BASADA EN FORRAJE SOBRE EL MOMENTO ÓPTIMO DE BENEFICIO DE CUYES
}

\section{Effect of the Inclusion of Barley Grain and Sunflower Seed in a Forage Diet on the Optimum Culling Age of Guinea Pigs}

\author{
Patricia Lozada P. ${ }^{1}$, Ronald Jiménez A. ${ }^{1,2}$, Felipe San Martín H. ${ }^{3}$, \\ Amparo Huamán C. ${ }^{1}$
}

\section{Resumen}

\begin{abstract}
El estudio evaluó el efecto de la suplementación energética sobre el momento óptimo económico de beneficio de cuyes en la Sierra peruana. Se utilizaron 200 cuyes machos de 4 semanas de edad, distribuidos en cuatro tratamientos por 13 semanas: forraje ad libitum (T0), forraje ad libitum más $10 \mathrm{~g}$ de cebada grano/animal (T1), forraje ad libitum más $7 \mathrm{~g}$ de semilla de girasol/animal (T2), y forraje ad libitum más $5 \mathrm{~g}$ de cebada grano y $3.5 \mathrm{~g}$ de semilla de girasol/animal (T3). Se empleó un diseño de bloques completos al azar, con el peso inicial como bloque, y 5 unidades experimentales de 10 animales por unidad. La suplementación energética mejoró significativamente la ganancia de peso y la conversión alimenticia $(\mathrm{p}<0.05)$; donde la combinación de granos girasol y cebada (T3) tienen un efecto adicional sobre la ganancia de peso, y la inclusión de girasol (T2) sobre la conversión alimenticia $(\mathrm{p}<0.05)$. La suplementación energética no tuvo efecto sobre el costo de producción, relación beneficio costo, ni edad óptima económica de beneficio.
\end{abstract}

Palabras clave: cobayo, energía, dieta, cebada grano, semilla de girasol, forraje, edad de beneficio

\section{Abstract}

The study evaluated the effect of the energy supplementation on the optimal culling age of guinea pigs (Cavia porcellus) in the Peruvian highlands. Two hundred guinea pigs of 4 weeks old were distributed in fours treatments during 13 weeks: forage $\mathrm{ad}$ libitum (T0), forage ad libitum plus $10 \mathrm{~g}$ barley grain/animal (T1), forage ad libitum plus $7 \mathrm{~g}$ sunflower seed/animal (T2), and forage ad libitum plus $5 \mathrm{~g}$ barley grain and $3.5 \mathrm{~g}$ sunflower seed/animal (T3). A randomized complete block design, with initial live weight

\footnotetext{
${ }^{1}$ Estación del Centro de Investigación IVITA - El Mantaro, ${ }^{3}$ Laboratorio de Bioquímica, Nutrición y Alimentación Animal, Facultad de Medicina Veterinaria, Universidad Nacional Mayor de San Marcos, Lima

${ }^{2}$ E-mail: rjimeneza@unmsm.edu.pe
} 
defining the block, and 5 experimental units of 10 animals each, was applied. The energy supplementation improved body weight gain and feed conversion index $(p<0.05)$, whereas the combination of sunflower seed and barley grain had an additional effect on body weight gain (T3) and the inclusion of sunflower (T2) on feed conversion index ( $\mathrm{p}<0.05)$. The energy supplementation had no effect on production cost, benefit/cost ratio or optimal culling age.

Key words: guinea pig, energy, diet, barley grain, sunflower seed, forage, culling age

\section{INTRODUCCIÓN}

Los sistemas de producción de cuyes en el Valle del Mantaro se sustentan principalmente en el suministro de forrajes y rastrojos. Se emplea mayormente alfalfa, así como como rye grass italiano, trébol rojo, avena y cebada (INIA, 1995). Sin embargo, bajo este tipo de alimentación tradicional, la energía es un componente dietético crítico pues, aunque el cuy puede consumir hasta el $40 \%$ de su peso vivo en forraje fresco, la capacidad del tracto gastrointestinal es limitada, siendo el volumen de forraje consumido insuficiente para cubrir sus requerimientos (Cheeke, 1995; Gómez y Vergara, 1995), surgiendo la necesidad de suplir la dieta con insumos de alta densidad energética.

Estudios en dietas de cuyes indican que el índice de conversión alimenticia (ICA) y la ganancia de peso mejoran con mayores niveles energéticos en la dieta, pues responden eficientemente al suministro de energía (Valer et al., 1986; Liza y Lozano, 1988; Santos y Cook, 1994; Jiménez, 2000). La disponibilidad de subproductos agrícolas y de granos convierte a estos insumos en una alternativa útil para que, a través de la suplementación en pequeñas cantidades, puedan cubrir el déficit energético de una dieta basada exclusivamente en forraje.

El momento óptimo económico de beneficio de cuyes es aquel momento donde la valorización del incremento en peso vivo del cuy por unidad de alimento (producto marginal) se iguala al costo del alimento. Estudios previos señalan que la suplementación con afrechillo a una dieta de solo alfalfa puede acortar la edad óptima de beneficio en una semana (Jiménez et al., 2000).

El presente estudio propone la alternativa de mejorar la alimentación de cuyes en condiciones de la Sierra peruana, adicionando insumos energéticos de disponibilidad local como cebada y semilla de girasol, cuya combinación suplementada a una dieta forrajera daría lugar a una mejor respuesta nutricional, una mayor ganancia de peso y, consecuentemente, una menor edad óptima económica de beneficio en cuyes.

\section{Materiales y Métodos}

El estudio se realizó en la Estación Experimental El Mantaro del Instituto Veterinario de Investigaciones Tropicales y de Altura, Facultad de Medicina Veterinaria, Universidad Nacional Mayor de San Marcos, ubicada en el valle del Mantaro, Junín. Tuvo una duración de 13 semanas, periodo que abarcó entre diciembre de 2005 a marzo de 2006.

Se utilizaron 200 cuyes machos tipo 1 mejorados, caracterizados por el pelo corto y lacio, y mejorados mediante selección por ganancia de peso. Los animales tenían cuatro semanas de edad y se distribuyeron en cuatro tratamientos bajo un diseño de Bloques Completos al Azar, donde los bloques fueron los rangos de peso inicial: A (300-500), B (501-560), C (561-620), D (621-660) y E 
(661-700 g), empleándose una unidad experimental por bloque dentro de tratamientos. Cada unidad experimental correspondió a una poza con 10 animales.

Los animales de los cuatro tratamientos recibieron una dieta a base de forraje fresco ad libitum, dando lugar a tres raciones de suplemento isoenergéticas, equivalente a 34 kcal ED de energía digestible, y un tratamiento control. El tratamiento T1 recibió, además, $10 \mathrm{~g}$ de grano de cebada entera por animal, el T2 se suplementó con 7 g/animal de semilla de girasol entera, el T3 se suplementó con $5 \mathrm{~g}$ de grano de cebada y $3.5 \mathrm{~g} /$ animal de semilla de girasol entera entera, en tanto que T0 no fue suplementado.

Se utilizó alfalfa variedad California 55 y una asociación de trébol rojo variedad Quinequelli y rye grass italiano variedad Belinda como fuentes forrajeras. El forraje verde fue pesado diariamente $\mathrm{y}$ ofrecido tres veces al día (8:00, 12:00, 16:00) a una razón inicial de $300 \mathrm{~g} / \mathrm{animal}$, con un aporte energético aproximado de $169 \mathrm{kcal}$ de energía digestible, cantidad que se incrementó según el consumo, manteniendo un incremento en paralelo del aporte energético para los tratamientos con suplementación. El suplemento se ofreció una vez al día (09:00). Los animales se pesaron semanalmente antes del suministro de alimentos.

Para calcular el consumo se registró diariamente el peso del forraje verde ofrecido y semanalmente el peso del forraje rechazado. Después de cada pesada se tomó una submuestra para determinación de materia seca, cuyo proceso fue el de desecación en estufa con aire circulante a $60{ }^{\circ} \mathrm{C}$ por 48 horas. El consumo se calculó por semana, siendo la diferencia entre alimento ofrecido y rechazado en base seca. La estimación del consumo de suplementos tuvo un procedimiento similar. El consumo de materia seca (MS) por animal se estimó al dividir el consumo total de la poza entre el número de animales en la poza.
La ganancia de peso semanal se obtuvo por diferencia entre los pesos promedios registrados al inicio y final de cada semana. Los datos de consumo y peso semanal fueron necesarios para la estimación del momento óptimo económico de beneficio; sin embargo, también se estimaron valores diarios para estas variables, pues son válidos para fines de comparación con otros estudios. La conversión alimenticia se calculó en base al consumo de materia seca sobre la ganancia de peso.

Se determinó el costo de producción unitario en base al cálculo detallado y anualizado de costos fijos y costos variables, cuyo total se dividió entra la cantidad de cuyes aptos para venta obtenidos por año. La estimación del cociente o ratio beneficio/costo consideró como beneficio al precio del kilo de peso vivo del cuy, equivalente a S/. 10.00 y como costo al costo unitario. El momento óptimo económico de beneficio se determinó mediante el método descrito por Bautista (1974).

Se empleó el análisis de varianza para evaluar las variables de ganancia de peso diaria, consumo de MS, conversión alimenticia, costo de producción y relación beneficio/costo, y la prueba de Duncan para determinar diferencias estadísticas entre tratamientos $(\mathrm{p}<0.05)$.

\section{Resultados y Discusión}

La suplementación incrementa significativamente la ganancia de peso, siendo la respuesta mayor cuando se combinan insumos que poseen diferentes fuentes energéticas, como en el caso de los granos de cebada y girasol (Cuadro 1) que aportan almidón y lípidos, respectivamente.

Cuando la producción de cuyes iniciaba su desarrollo tecnológico, las primeras evaluaciones de alimentos forrajeros (Paredes, 1972; Arroyo, 1974) obtenían bajas ganan- 
Cuadro 1. Parámetros productivos y económicos en cuyes según el tipo de dieta isocalórica ${ }^{1}$

\begin{tabular}{lccccc}
\hline \multirow{2}{*}{ Parámetro evaluado } & \multicolumn{4}{c}{ Tratamientos } \\
\cline { 2 - 5 } & T 0 & T1 & T2 & T3 & \\
\hline Ganancia de peso diaria (g) & $6.40^{\mathrm{a}}$ & $7.70^{\mathrm{b}}$ & $8.14^{\mathrm{b}}$ & $8.68^{\mathrm{c}}$ & 0.31 \\
Consumo de MS (g) & $68.98^{\mathrm{a}}$ & $72.46^{\mathrm{b}}$ & $69.44^{\mathrm{a}}$ & $68.88^{\mathrm{a}}$ & 1.196 \\
Consumo de MS (forraje) (g) & 68.98 & 63.53 & 63.51 & 61.37 & - \\
Consumo de MS (concentrado) (g) & - & 8.93 & 5.93 & 7.51 & - \\
Conversión alimenticia & $10.88^{\mathrm{a}}$ & $9.48^{\mathrm{b}}$ & $8.52^{\mathrm{c}}$ & $8.04^{\mathrm{c}}$ & 0.467 \\
Costo de producción (S/.) & $4.60^{\mathrm{a}}$ & $4.59^{\mathrm{a}}$ & $4.87^{\mathrm{a}}$ & $4.59^{\mathrm{a}}$ & 0.121 \\
Relación beneficio / costo & $2.19^{\mathrm{a}}$ & $2.19^{\mathrm{a}}$ & $2.07^{\mathrm{a}}$ & $2.19^{\mathrm{a}}$ & 0.023 \\
Momento óptimo económico de beneficio & $11.36^{\mathrm{a}}$ & $14.79^{\mathrm{a}}$ & $12.25^{\mathrm{a}}$ & $11.29^{\mathrm{a}}$ & 18.803 \\
(semanas) & & & & & \\
\hline
\end{tabular}

${ }^{1}$ TO: forraje; T1: forraje + cebada grano; $\mathrm{T2}$ : forraje + semilla de girasol; T3: forraje + cebada grano + semilla de girasol

2 Cuadrado medio del error

a,b,c Superíndices diferentes dentro de filas indican diferencia estadística $(p \varangle 0.05)$

Cuadro 2. Edad óptima económica de beneficio de cuyes según el tipo de dieta isocalórica ${ }^{1}$

\begin{tabular}{ccccc}
\hline \multirow{2}{*}{ Bloques } & \multicolumn{4}{c}{ Momento óptimo económico de beneficio (en semanas) } \\
\cline { 2 - 5 } & T0 & T1 & T2 & T3 \\
\hline I & 8.82 & 21.25 & 7.55 & 12.47 \\
II & 9.09 & 14.90 & 17.04 & 13.68 \\
III & 13.17 & 20.54 & 13.36 & 12.54 \\
IV & 13.02 & 13.30 & 9.70 & 8.49 \\
V & 12.69 & 3.94 & 13.59 & 9.26 \\
\hline Promedio & 11.36 & 14.79 & 12.25 & 11.29 \\
\hline
\end{tabular}

${ }^{1}$ TO: forraje; T1: forraje +cebada grano; T2: forraje +semilla de girasol; T3: forraje + cebada grano + semilla de girasol

cias de peso. Estudios posteriores mejoraron la ganancia de peso no solo por la mejora genética sino también por el uso de suplementos concentrados (Vásquez et al., 1987; Espinoza, 1991), que incluso superaron los resultados hallados en el presente estudio (Jiménez et al., 2000; Matos et al., 2003).
La suplementación con cebada incrementa significativamente el consumo total debido a un efecto de tipo aditivo sustitutivo, mientras que la inclusión de girasol grano solo ejerce un efecto sustitutivo en el consumo de forraje (Cuadro 1). Estudios previos (Huacho, 1971; Arroyo, 1974; Arroyo et 
Cuadro 3. Estudios previos sobre edad óptima económica de beneficio en cuyes

\begin{tabular}{lcl}
\hline Característica del estudio & $\begin{array}{c}\text { Edad óptima de } \\
\text { beneficio (semanas) }\end{array}$ & Referencia \\
\hline Macho con alimento concentrado & 9.78 & Bautista (1974) \\
Hembra con alimento concentrado & 9.97 & $\begin{array}{l}\text { Escobar y Tapia } \\
\text { Macho alimen tado con alfalfa + maíz + sal }\end{array}$ \\
mineral & 12.7 & Zaldivar et al. \\
Línea Perú & 8 & $(1988)$ \\
Línea Inti & 10 & Saavedra y \\
Alimentado con forraje & 9 & Espinoza (1988) \\
Alimentado con forraje + concentrado & 7 & De la Peña y \\
Castrado con forraje + cebada + sal mineral & 13.04 & Taipe (1991) \\
Macho con alfalfa & 12.87 & \\
Hembra con alfalfa & 12.94 & Jiménez et al. \\
Macho con alfalfa + afrechillo & 11.75 & (2000) \\
Hembra con alfalfa + afrechillo & 11.99 & \\
\hline
\end{tabular}

al., 1974; Valer et al., 1986) refieren menor consumo respecto al presente estudio; probablemente porque se suministró alimento a discreción, además de emplearse animales de mayor peso.

La suplementación contribuye a mejorar la conversión alimenticia y cuando el insumo posee mayor densidad energética, como el caso del grano de girasol, se reduce aun más el índice de conversión (Cuadro 1). Estos resultados coinciden con otros estudios (Arroyo et al., 1974; Valer et al., 1986; Jiménez et al., 2000) que también mejoraron la conversión tras la inclusión de un suplemento energético y concentrado a una dieta basada en alfalfa verde.

El costo de producción y el ratio beneficio costo no mejoran por efecto de la suplementación (Cuadro 1). Sin embargo, si la suplementación mejora la ganancia de peso, el cuy puede alcanzar el peso de mercado en un menor tiempo; acortándose el periodo de permanencia en granja para beneficio del productor.
No se evidencia un efecto de las dietas isocalóricas sobre el momento óptimo económico de beneficio, pues los resultados no muestran una tendencia definida y poseen alta variabilidad dentro de tratamientos (Cuadro 1 y 2). Uno de los factores más importantes de esta variabilidad es que el cuy mostró un comportamiento variable en el consumo de materia seca, probablemente influenciado por el nivel de humedad del forraje, el cual no siempre fue creciente de una semana a otra, dando lugar a que las ecuaciones de regresión presenten un mayor error. Los valores promedio refieren que ante una dieta de solo forraje o suplementada con un concentrado energético, la edad óptima de beneficio se encuentra entre la semana 11 y 12 (Cuadro 2).

Resultados de estudios previos que emplearon la misma metodología, encontraron que la suplementación con concentrado acorta la edad óptima económica de beneficio (Cuadro 3). No obstante, se aprecia variabilidad entre resultados, con un amplio rango ( 7 a 24 semanas) de edades óptimas de beneficio. 
Obviamente en estos estudios hay factores adicionales que deben tenerse en consideración, especialmente el genético y el alimenticio.

Existen otros métodos de estimación de la edad óptima de beneficio o saca con procedimientos más sencillos (Aliaga et al., 2009), cuya precisión de estimación sería conveniente medirlo en comparación al método empleado en este estudio y algún referente de alta precisión.

\section{Conclusiones}

- La inclusión de suplementos energéticos a dietas de forraje verde en cuyes no modificó la edad óptima económica de beneficio.

- La suplementación energética mejoró la ganancia de peso y conversión alimenticia.

- La suplementación con grano de girasol respecto al grano de cebada dio una mejor respuesta en conversión alimenticia.

\section{Literatura Citada}

1. Aliaga L, Moncayo $R$, Rico $E$, Caycedo A. 2009. Producción de cuyes. Lima: Fondo Editorial de la Universidad Católica Sedes Sapientiae. 808 p.

2. Arroyo O. 1974. Uso de la alfalfa verde y el heno de alfalfa en el engorde de cuyes. En: Investigación en cuyes. Vol I. Huancayo: Universidad Nacional del Centro del Perú. p 23-28.

3. Arroyo O, Aliaga L, Knutzen E. 1974. Evaluación de la vitamina $\mathrm{C}$ y concentrado como sustitución del forraje en cobayos destetados. En: Investigación en cuyes. Vol I. Huancayo: Universidad Nacional del Centro del Perú. p 20-22.

4. Bautista A. 1974. Determinación de la edad óptima de comercialización y selec- ción de cuyes (Cavia porcellus). Tesis de Ingeniero Zootecnista. Lima. Universidad Nacional Agraria La Molina. 59 p.

5. Cheeke P. 1995. Alimentación y nutrición del conejo. Madrid: Acribia. 444 p.

6. De la Peña N, Taipe H. 1991. El óptimo económico en el beneficio de cuyes mejorados no enteros engordados con tres raciones en zona quechua (2450 msnm) Ayacucho. En: XIV Reunión APPA. Cerro de Pasco: Asociación Peruana de Producción Animal.

7. Escobar F, Tapia H. 1985. Determinación del óptimo económico de saca de cuyes mejorados. En: VIII Reunión APPA. Huancayo: Asociación Peruana de Producción Animal.

8. Espinoza T. 1991. Engorde de cuyes castrados a base de concentrado y forraje. En: XIV Reunión APPA. Cerro de Pasco: Asociación Peruana de Producción Animal.

9. Gómez C, Vergara YV. 1995. Fundamentos de la nutrición y alimentación. En: Serie Guía Didáctica: Crianza de Cuyes. Lima. INIA-DGTT. p 27-35.

10. Huacho C. 1971. Comparativo de cuatro raciones para cobayos en crecimiento. Tesis de Ingeniero Zootecnista. Lima. Universidad Nacional Agraria La Molina. $77 \mathrm{p}$.

11. [INIA] Instituto Nacional de Investigación Agraria. 1995. Proyecto Sistemas de Producción de Cuyes. Lima. INIA. $85 \mathrm{p}$.

12. Jiménez R. 2000. Determinación del momento óptimo económico de beneficio de cuyes del C.I. IVITA -Huancayo alimentados con alfalfa vs. una alimentación con afrechillo. Tesis de Médico Veterinario. Lima: Facultad de Medicina Veterinaria, Univ Nacional Mayor de San Marcos. 59 p.

13. Jiménez R, Bojórquez C, San Martín F, Carcelén F, Pérez A. 2000. Determinación del momento óptimo económico de beneficio de cuyes alimentados con alfalfa vs. una suplementación con afrechillo. Rev Inv Vet, Perú 11(1): 45-51. 
14. Liza Z, Lozano E. 1988. Niveles crecientes de afrecho de algarrobo en el crecimiento-acabado de cuyes. En: XI Reunión APPA. Piura: Asociación Peruana de Producción Animal.

15. Mattos J, Chauca L, San Martín F. 2003. Uso del ensilado biológico de pescado en la alimentación de cuyes mejorados. Rev Inv Vet, Perú 14(2): 89-96.

16. Paredes L. 1972. Utilización de diferentes niveles de alfalfa en la alimentación de cuyes. Tesis de Ingeniero Zootecnista. Lima. Universidad Nacional Agraria La Molina. 61 p.

17. Saavedra E, Espinoza F. 1988. Análisis para determinar el momento óptimo de saca de cuyes sometidos a engorde. En: XI Reunión APPA. Piura: Asociación Peruana de Producción Animal.

18. Santos D, Cook F. 1988. Engorde de cuyes mejorados con alfalfa verde, con centrado comercial y cebada remojada. En: XI Reunión APPA. Piura: Asociación Peruana de Producción Animal.

19. Valer A, Blas C, Taipe H. 1986. Engorde de cuyes machos mejorados a base de dos raciones y evaluaciones de la carcasa. En: IX Reunión APPA. Tingo María: Asociación Peruana de Producción Animal.

20. Vásquez G, Lozano E, Guerrero J. 1987. Crecimiento - engorde de cuyes alimentados con chope (Cryptorcarpus pyriformis, HBK), alfalfa y concentrado. En: X Reunión APPA. Puno: Asociación Peruana de Producción Animal.

21. Zaldivar M, Chauca L, Gutiérrez N, Ganoza V. 1988. Evaluación económica de cuatro líneas de cuyes (Cavia porcellus). En: XI Reunión APPA. Piura: Asociación Peruana de Producción Animal. 